\title{
Adaptive Joint Self-Interference Cancellation and Equalization for Space-Time Coded Bi-Directional Relaying Networks
}

\author{
Jeong-Min Choi*, Jae-Shin Han*, and Jong-Soo Seo* \\ ${ }^{*}$ Department of Electrical and Electronic Engineering, Yonsei University \\ 50 Yonsei-ro, Seodaemun-gu, Seoul, 120-749, Republic of Korea \\ choi_jm@yonsei.ac.kr, hjs4015@yonsei.ac.kr, jsseo@yonsei.ac.kr
}

\begin{abstract}
In this paper, we propose an adaptive receiver for joint self-interference cancellation and equalization in an amplifyand-forward (AF) bi-directional relaying network. In particular, we develop an efficient receiver that does not require the complex matrix inversions. The performance of the proposed scheme is demonstrated by computer simulations.
\end{abstract}

Index Terms-Adaptive receiver, bi-directional relaying network, equalization, joint detection, self-interference cancellation.

\section{INTRODUCTION}

Bi-directional relaying networks have attracted lots of attention owing to its ability to circumvent the loss of spectral efficiency in one-way relaying networks [1]-[4]. Further, various relaying protocols have been combined with space-time block codes (STBCs) to further improve the spectral efficiency [2]-[3]. Although many researches related to bi-directional relaying networks have been established, the issues on the receiver design have gained relatively little attention in the literature. One of the main reasons for this is due to the common belief that such issues can be explained by the traditional point-to-point communication or one-way relaying systems. However, in bidirectional relaying networks, such issues would be different from those of such systems due to the several reasons: First of all, in bi-directional relaying networks, the amount of system overheads increases compared to the oneway relaying protocol due to the separate processing of selfinterference cancellation and data detection, i.e., the required number of links to be estimated increases. In particular, in frequency selective fading channels, more challenges on such issues will appear since a long training sequence (TS) should be used for the reliable channel estimation [5]. Moreover, if the nodes have multiple antennas, the minimum required length of TS increases due to the increasing number of propagation links [5]. Secondly, because of the two phase transmissions, if STBC is employed, the block size must be shorter than a quarter of the channel coherence time to prevent the performance degradation caused by the time selectivity. It further reduces spectral efficiency since the ratio of pure payload to overhead decreases. As a result, the improvement of spectral efficiency, main advantage of a bi-directional relaying compared to oneway relaying, could not be maximized. Finally, more importantly, additional overhead signaling is further required at the relay node during the second phase in order to estimate channel state of relay-to-destination link which is necessary to compute covariance matrix of the effective noise used in minimum mean square error (MMSE) equalization processing. This procedure makes the frame structure complex and reduces spectral efficiency. To avoid these unfavorable effects, the approximate approach can be applied to the computation of covariance matrix [6]. However, it leads to the performance degradation significantly.

In this paper, as an alternative to the channel state information (CSI)-based receiver, we develop an adaptive joint receiver that does not require CSI. To the best of our knowledge, it is the first attempt to apply adaptive scheme to bi-directional relaying network. The presented joint receiver requires no complex matrix inversion and can reduce system overhead compared to CSI-based receiver as well as provides MMSElike performance. Thus, it can be simply implemented and improve the spectral efficiency with high quality of reception in slow fading channels.

Notations: $|\cdot|,(\cdot)^{*},(\cdot)^{T}$ and $(\cdot)^{\dagger}$ denote the absolute value, complex conjugate, transpose, and Hermitian transpose operations, respectively. $\otimes, \operatorname{tr}(\cdot)$, and $E[\cdot]$ stand for the Kronecker delta product, a trace of a matrix, and expectation, respectively. (.) $\bmod N$ denotes the modulo- $N$ operation. $\mathbf{0}_{M \times K}$ and $\mathbf{I}_{M}$ denote $M \times K$ all-zero matrix and identity matrix of size $M \times M$, respectively. $\mathbf{Q}$ denotes the $N \times N$ discrete Fourier transform (DFT) matrix. $\mathbf{P}$ denotes an $N \times N$ permutation matrix and it performs a reversed cyclic shift. For instance, given $\mathbf{a}=[a(0), \ldots, a(N-1)]^{T}, \mathbf{P a}=[a(N-1), \ldots, a(0)]^{T}$.

\section{TR-STBC BI-DiRECTIONAL RELAYING NETWORKS}

\section{A. System Model}

Let us consider a bi-directional relaying network consisting of two source terminals $\left(\mathbb{T}_{1}, \mathbb{T}_{2}\right)$ and a single relay $(\mathbb{R})$ as depicted in Fig. 1. At this time, it is assumed that $\mathbb{T}_{1}$ and $\mathbb{T}_{2}$ are equipped with two antennas while $\mathbb{R}$ has a single antenna [3]. In Fig. 1, we define $\mathbf{x}_{i}^{k}=\left[x_{i}^{k}(0), \ldots, x_{i}^{k}(N-1)\right]^{T}$ of size $N \times 1(i=1,2)$ as the $k$ th data block transmitted from the $i$ th antenna of $\mathbb{T}_{1}$ to $\mathbb{R}$. Similarly, $\mathbf{d}_{i}^{k}=\left[d_{i}^{k}(0), \ldots, d_{i}^{k}(N-1)\right]^{T}$ of size $N \times 1$ represents the transmit signal from the $i$ th antenna of $\mathbb{T}_{2}$ to $\mathbb{R}$. In addition, according to the encoding rule of a time-reversal STBC (TR-STBC) [7], the $k+1$ th 


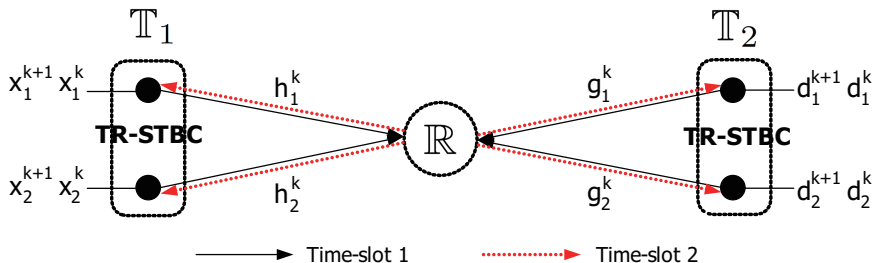

Fig. 1. TR-STBC bi-directional relaying network over frequency-selective fading channels.

data blocks to be transmitted are defined as $\mathbf{x}_{1}^{k+1}=\mathbf{P} \mathbf{x}_{2}^{k *}$, $\mathbf{x}_{2}^{k+1}=-\mathbf{P} \mathbf{x}_{1}^{k *}, \mathbf{d}_{1}^{k+1}=\mathbf{P d}_{2}^{k *}$, and $\mathbf{d}_{2}^{k+1}=-\mathbf{P d}_{1}^{k *}$. We also define the CIR of the propagation link between the $i$ th antenna of $\mathbb{T}_{1}$ and $\mathbb{R}$ as $\mathbf{h}_{i}^{k}=\left[h_{i}^{k}(0), \ldots, h_{i}^{k}\left(L_{i}-1\right)\right]^{T}$ with $\sum_{l_{i}-1}^{L_{i}} E\left[\left|h_{i}^{k}(l)\right|^{2}\right]=1 . \mathbf{g}_{i}^{k}=\left[g_{i}^{k}(0), \ldots, g_{i}^{k}\left(P_{i}-1\right)\right]^{T}$ with $\sum_{l}^{P_{i}-1} E\left[\left|g_{i}^{k}(l)\right|^{2}\right]=1$ is the CIR of the link between the $i$ th antenna of $\mathbb{T}_{2}$ and $\mathbb{R}$. Here, $L_{i}$ and $P_{i}$ denote the corresponding channel lengths. It is also assumed that the entries of $\mathbf{h}_{i}^{k}$ and $\mathbf{g}_{i}^{k}$ are statistically independent of each other. Moreover, let $\mathbf{H}_{i}^{k}$ and $\mathbf{G}_{i}^{k}$ be $N \times N$ circulant matrices, whose entries are given by $\left[\mathbf{H}_{i}^{k}\right]_{m, l}=\mathbf{h}_{i}^{k}((m-l) \bmod N)$ and $\left[\mathbf{G}_{i}^{k}\right]_{m, l}=\mathbf{g}_{i}^{k}((m-l) \bmod N)$, respectively [7]. We consider a block fading wireless channels where the channel remains constant over blocks, and changes from block to block slowly.

\section{B. Relaying Protocol}

In Fig. 1, each block transmission is completed during two time-slots [2]-[3]. In the first time-slot, both $\mathbb{T}_{1}$ and $\mathbb{T}_{2}$ transmit signals to $\mathbb{R}$, simultaneously. Before the transmission, we notice that a cyclic-prefix (CP) of length- $\nu$ is appended in front of the data block and it satisfies the condition of $\nu \geq \max \left(L_{1}, L_{2}, P_{1}, P_{2}\right)-1$. At the receiver side of $\mathbb{R}$, the relay removes the $\mathrm{CP}$ and its resulting signal is given by

$$
\mathbf{y}_{R}^{k}=\sum_{l=1}^{2}\left(\sqrt{E_{1} / 2} \mathbf{H}_{l}^{k} \mathbf{x}_{l}^{k}+\sqrt{E_{2} / 2} \mathbf{G}_{l}^{k} \mathbf{d}_{l}^{k}\right)+\mathbf{n}_{R}^{k}
$$

where $E_{i}$ denotes the average transmit power of $\mathbb{T}_{i}(i=1,2)$, and $\mathbf{n}_{R}^{k}$ denotes the additive white Gaussian noise (AWGN) vector whose entries are statistically zero-mean and variance $\sigma_{n R}^{2}$. Then, in order to meet the power constraint of $E_{R}$ at the transmit side of $\mathbb{R}$, the relay scales $\mathbf{y}_{R}^{k}$ with $\sqrt{E_{R} /\left(E_{1}+E_{2}+\sigma_{n R}^{2}\right)}$, of which output signal $\mathbf{r}^{k}$ can be expressed as

$$
\mathbf{r}^{k}=\gamma_{1}\left(\mathbf{H}_{1}^{k} \mathbf{x}_{1}^{k}+\mathbf{H}_{2}^{k} \mathbf{x}_{2}^{k}\right)+\gamma_{2}\left(\mathbf{G}_{1}^{k} \mathbf{d}_{1}^{k}+\mathbf{G}_{2}^{k} \mathbf{d}_{2}^{k}\right)+\gamma_{3} \mathbf{n}_{R}^{k}
$$

where the scaling factors $\gamma_{1}, \quad \gamma_{2}, \quad$ and $\gamma_{3}$ are defined as $\gamma_{1}=\sqrt{E_{1} E_{R} /\left(2\left(E_{1}+E_{2}+\sigma_{n R}^{2}\right)\right)}$, $\gamma_{2}=\sqrt{E_{2} E_{R} /\left(2\left(E_{1}+E_{2}+\sigma_{n R}^{2}\right)\right)}$, and $\gamma_{3}=$ $\sqrt{E_{R} /\left(E_{1}+E_{2}+\sigma_{n R}^{2}\right)}$, respectively. In the second time-slot, $\mathbb{R}$ broadcasts the signal to $\mathbb{T}_{1}$ and $\mathbb{T}_{2}$ after re-inserting the $\mathrm{CP}$ into $\mathbf{r}^{k}$. Here, we also notice that the receiver operation and structure of $\mathbb{T}_{1}$ and $\mathbb{T}_{2}$ are identical to each other [1]-[4]. Hence, without loss of generality, we will only describe the signal reconstruction at $\mathbb{T}_{1}$ in the rest of this letter. As a result, considering $\mathbf{H}_{p}^{k}$ effect into $\mathbf{r}^{k}$ and discarding the $\mathrm{CP}$, the received signal at the $p$ th antenna of $\mathbb{T}_{1}$ (for $p=1,2$ ) can be expressed as

$$
\mathbf{y}_{p}^{k}=\gamma_{1}\left(\mathbf{H}_{p 1}^{k} \mathbf{x}_{1}^{k}+\mathbf{H}_{p 2}^{k} \mathbf{x}_{2}^{k}\right)+\gamma_{2}\left(\tilde{\mathbf{H}}_{p 1}^{k} \mathbf{d}_{1}^{k}+\tilde{\mathbf{H}}_{p 2}^{k} \mathbf{d}_{2}^{k}\right)+\boldsymbol{\Delta}_{p}^{k}
$$

where $\mathbf{H}_{p q}^{k}=\mathbf{H}_{p}^{k} \mathbf{H}_{q}^{k}$ and $\tilde{\mathbf{H}}_{p q}^{k}=\mathbf{H}_{p}^{k} \mathbf{G}_{q}^{k}(q=1,2)$. Here, it is assumed that the CIRs remain constant during two timeslots. This assumption does not lose the generality since the length of data block is determined to preserve good TRSTBC decoupling performance and the adaptive filter generally works in slow fading environments [8]-[9]. In (3), $\Delta_{p}^{k}=$ $\gamma_{3} \mathbf{H}_{p}^{k} \mathbf{n}_{R}^{k}+\mathbf{n}_{T_{1}, p}^{k}$ denotes the effective noise vector, where $\mathbf{n}_{T_{1}, p}^{k}$ denotes AWGN vector generated in the $p$ th antenna of $\mathbb{T}_{1}$ with each entry having zero-mean and variance $\sigma_{n}^{2}$, and $E\left[\boldsymbol{\Delta}_{p}^{k} \boldsymbol{\Delta}_{p}^{k \dagger} \mid \mathbf{h}_{p}^{k}\right]=\left(\gamma_{3}^{2} \sigma_{n R}^{2} \sum_{l=0}^{L_{p}-1}\left|h_{p}^{k}(l)\right|^{2}+\sigma_{n}^{2}\right) \mathbf{I}_{N} \triangleq \sigma_{\Delta_{p}}^{2} \mathbf{I}_{N}$.

\section{The Proposed Adaptive Receiver Scheme}

In this section, we first derive the frequency-domain CSIbased receiver composing of separate processing of selfinterference cancellation and MMSE equalization. Then, from the mathematical formulation of CSI-based receiver, we derive an efficient adaptive receiver that jointly performs selfinterference cancellation, TR-STBC decoupling and channel equalization.

Before the presentation, let us first assume that the CIRs are constant over two consecutive blocks for a complete TR-STBC decoupling, i.e., $\mathbf{H}_{p q}^{k}=\mathbf{H}_{p q}^{k+1} \triangleq \mathbf{H}_{p q}$ and $\tilde{\mathbf{H}}_{p q}^{k}=\tilde{\mathbf{H}}_{p q}^{k+1} \triangleq$ $\tilde{\mathbf{H}}_{p q}$ in (3). Again, it should be noted that this assumption does not lose the generality. It is also assumed that the related CIRs are perfectly known to the receiver. As a result, using the encoding rule of TR-STBC [7] and taking DFT processing $\mathbf{Q}$ into (3), the received frequency-domain signals at the $p$ th antenna of $\mathbb{T}_{1}$ over the blocks $k$ and $k+1$ can be written as follows

$$
\begin{aligned}
\mathcal{Y}_{p}^{k} & =\boldsymbol{\Sigma}_{p 1} \mathcal{X}_{1}^{k}+\boldsymbol{\Sigma}_{p 2} \mathcal{X}_{2}^{k}+\boldsymbol{\Lambda}_{p 1} \mathcal{D}_{1}^{k}+\boldsymbol{\Lambda}_{p 2} \mathcal{D}_{2}^{k}+\mathbf{Q} \boldsymbol{\Delta}_{p}^{k}, \\
\overline{\mathcal{Y}}_{p}^{k+1} & =-\boldsymbol{\Sigma}_{p 2}^{\dagger} \mathcal{X}_{1}^{k}+\boldsymbol{\Sigma}_{p 1}^{\dagger} \mathcal{X}_{2}^{k}-\boldsymbol{\Lambda}_{p 2}^{\dagger} \mathcal{D}_{1}^{k}+\boldsymbol{\Lambda}_{p 1}^{\dagger} \mathcal{D}_{2}^{k}+\mathbf{Q} \overline{\boldsymbol{\Delta}}_{p}^{k+1}
\end{aligned}
$$

where $\mathcal{Y}_{p}^{k}=\mathbf{Q} \mathbf{y}_{p}^{k}, \overline{\mathcal{Y}}_{p}^{k+1}=\mathbf{Q P y}_{p}^{k+1 *}, \boldsymbol{\mathcal { X }}_{l}^{k}=\mathbf{Q} \mathbf{x}_{l}^{k}(l=$ $1,2), \mathcal{D}_{l}^{k}=\mathbf{Q d}_{l}^{k}$, and $\overline{\boldsymbol{\Delta}}_{p}^{k+1}=\mathbf{P} \boldsymbol{\Delta}_{p}^{k+1 *}$. In (4), $\boldsymbol{\Sigma}_{p q}=$ $\gamma_{1} \mathbf{Q} \mathbf{H}_{p q} \mathbf{Q}^{\dagger}, \boldsymbol{\Sigma}_{p q}^{\dagger}=\gamma_{1} \mathbf{Q} \mathbf{P} \mathbf{H}_{p q}^{*} \mathbf{P} \mathbf{Q}^{\dagger}, \boldsymbol{\Lambda}_{p q}=\gamma_{2} \mathbf{Q} \tilde{\mathbf{H}}_{p q} \mathbf{Q}^{\dagger}$, and $\boldsymbol{\Lambda}_{p q}^{\dagger}=\gamma_{2} \mathbf{Q P} \tilde{H}_{p q}^{*} \mathbf{P} \mathbf{Q}^{\dagger}$ denote the effective diagonal channel matrices [7].

\section{A. Self-Interference Cancellation}

To detect $\mathcal{D}_{l}^{k}(l=1,2)$ in (4), $\mathbb{T}_{1}$ first eliminates the selfinterference signals (knows as its own transmit signals) $\boldsymbol{\mathcal { X }}_{l}^{k}$ by assuming that the corresponding channel coefficients $\boldsymbol{\Sigma}_{p q}$ is known to $\mathbb{T}_{1}$. As a result, at the $p$ th branch (connected with $p$ th antenna), by subtracting $\boldsymbol{\Sigma}_{p 1} \mathcal{X}_{1}^{k}+\boldsymbol{\Sigma}_{p 2} \mathcal{X}_{2}^{k}$ from $\mathcal{Y}_{p}^{k}$ and $-\boldsymbol{\Sigma}_{p 2}^{\dagger} \mathcal{X}_{1}^{k}+\boldsymbol{\Sigma}_{p 1}^{\dagger} \mathcal{X}_{2}^{k}$ from $\overline{\mathcal{Y}}_{p}^{k+1}$ in (4), the outputs $\mathcal{Z}_{1}$ and $\mathcal{Z}_{2}$ at the respective branches can be written as an Alamouti-like form:

$$
\mathcal{Z}_{1}=\mathcal{M}_{1} \mathcal{D}+\Upsilon_{1}, \quad \mathcal{Z}_{2}=\mathcal{M}_{2} \mathcal{D}+\Upsilon_{2}
$$

where $\mathcal{D}=\left[\mathcal{D}_{1}^{k T}, \mathcal{D}_{2}^{k T}\right]^{T}, \mathbf{\Upsilon}_{p}=\left[\mathbf{Q} \boldsymbol{\Delta}_{p}^{k T}, \mathbf{Q} \bar{\Delta}_{p}^{k+1 T}\right]^{T}$, and the effective channel matrices $\mathcal{M}_{1}$ and $\mathcal{M}_{2}$ are

$$
\mathcal{M}_{1}=\left[\begin{array}{cc}
\boldsymbol{\Lambda}_{11} & \boldsymbol{\Lambda}_{12} \\
-\boldsymbol{\Lambda}_{12}^{\dagger} & \boldsymbol{\Lambda}_{11}^{\dagger}
\end{array}\right], \mathcal{M}_{2}=\left[\begin{array}{cc}
\boldsymbol{\Lambda}_{21} & \boldsymbol{\Lambda}_{22} \\
-\boldsymbol{\Lambda}_{22}^{\dagger} & \boldsymbol{\Lambda}_{21}^{\dagger}
\end{array}\right] \text {. }
$$




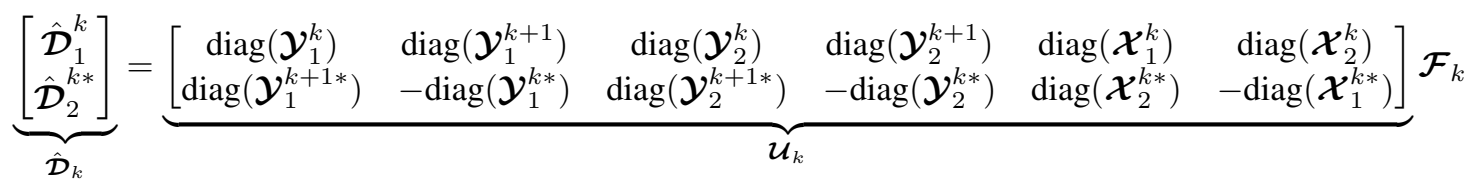

\section{B. MMSE Equalization}

For data decoding from the outputs of (5), MMSE equalization including TR-STBC decoupling is then accomplished. Multiplying $\mathcal{M}_{1}^{\dagger}$ to $\mathcal{Z}_{1}$ and $\mathcal{M}_{2}^{\dagger}$ to $\mathcal{Z}_{2}$, the outputs of TRSTBC decoupling are given, respectively, by

$$
\begin{aligned}
& \mathcal{M}_{1}^{\dagger} \mathcal{Z}_{1}=\left(\mathbf{I}_{2} \otimes\left(\left|\boldsymbol{\Lambda}_{11}\right|^{2}+\left|\boldsymbol{\Lambda}_{12}\right|^{2}\right)\right) \mathcal{D}+\mathcal{M}_{1}^{\dagger} \boldsymbol{\Upsilon}_{1}, \\
& \mathcal{M}_{2}^{\dagger} \mathcal{Z}_{2}=\left(\mathbf{I}_{2} \otimes\left(\left|\boldsymbol{\Lambda}_{21}\right|^{2}+\left|\boldsymbol{\Lambda}_{22}\right|^{2}\right)\right) \mathcal{D}+\mathcal{M}_{2}^{\dagger} \boldsymbol{\Upsilon}_{2}
\end{aligned}
$$

where it is noticed that it suffices to look into only one data block in the equalization process, since the effective channel gain matrices for both $\mathcal{D}_{1}^{k}$ and $\mathcal{D}_{2}^{k}$ are equal and the noise terms are identically distributed and statistically uncorrelated with each other. Therefore, without loss of generality, the detection is shown only for the case of $\mathcal{D}_{1}^{k}$. We also drop the block index $k$ and replace $\mathcal{D}_{1}^{k}$ with $\tilde{\mathcal{D}}$ for brevity. Stacking the output vectors of the respective TR-STBC decoupling in (7), we define the following equation

$$
\mathcal{S}=\Gamma \tilde{\mathcal{D}}+\boldsymbol{\omega}
$$

where $\boldsymbol{\Gamma}$ denotes the effective channel gain matrix defined as

$$
\boldsymbol{\Gamma}=\left[\begin{array}{l}
\boldsymbol{\Gamma}_{1} \\
\boldsymbol{\Gamma}_{2}
\end{array}\right]=\left[\begin{array}{l}
\left|\boldsymbol{\Lambda}_{11}\right|^{2}+\left|\boldsymbol{\Lambda}_{12}\right|^{2} \\
\left|\boldsymbol{\Lambda}_{21}\right|^{2}+\left|\boldsymbol{\Lambda}_{22}\right|^{2}
\end{array}\right]
$$

and $\boldsymbol{\omega}$ stands for the effective noise vector with the associate covariance matrix $\boldsymbol{\Phi}_{\omega}=E\left[\boldsymbol{\omega} \boldsymbol{\omega}^{\dagger}\right]$ being

$$
\boldsymbol{\Phi}_{\omega}=\left[\begin{array}{cc}
\gamma_{2}^{2} \sigma_{\Delta_{1}}^{2} \boldsymbol{\Gamma}_{1} & \boldsymbol{\epsilon}^{\dagger} \\
\boldsymbol{\epsilon} & \gamma_{2}^{2} \sigma_{\Delta_{2}}^{2} \boldsymbol{\Gamma}_{2}
\end{array}\right]
$$

where $\boldsymbol{\epsilon}=\gamma_{2}^{2} \gamma_{3}^{2} \sigma_{n R}^{2}\left(\boldsymbol{\Lambda}_{21}^{\dagger} \boldsymbol{\Lambda}_{11}+\boldsymbol{\Lambda}_{22} \boldsymbol{\Lambda}_{12}^{\dagger}\right)$. After the channel equalization, the output of MMSE equalization is transformed back to the time-domain. As a result, the error vector between the output of the equalizer and the true signal can be expressed as $\mathbf{e}=\mathbf{Q}^{\dagger} \mathcal{W} \mathcal{S}-\mathbf{Q}^{\dagger} \tilde{\mathcal{D}}$, where $\mathcal{W}=\left[\mathcal{W}_{1}, \mathcal{W}_{2}\right]$ denotes the MMSE equalization and its submatrices $\mathcal{W}_{1}$ and $\mathcal{W}_{2}$ are real-valued $N \times N$ diagonal matrices. From the orthogonal principle, $E\left[\left(\mathbf{Q}^{\dagger} \mathcal{W} \mathcal{S}-\mathbf{Q}^{\dagger} \tilde{\mathcal{D}}\right) \mathcal{S}^{\dagger}\right]=\mathbf{0}_{N \times N}, \mathcal{W}$ can be readily obtained as

$$
\mathcal{W}=\boldsymbol{\Gamma}^{\dagger}\left(\boldsymbol{\Gamma} \boldsymbol{\Gamma}^{\dagger}+\boldsymbol{\Phi}_{\omega}\right)^{-1}
$$

Moreover, the theoretical MSE given by $\operatorname{tr}\left(E\left[\mathbf{e e}^{\dagger}\right]\right) / N$ will be $\operatorname{tr}\left(\mathbf{I}_{N}-\left(\mathbf{I}_{N}+\boldsymbol{\Gamma}^{\dagger} \boldsymbol{\Phi}_{\omega}^{-1} \boldsymbol{\Gamma}\right)^{-1} \boldsymbol{\Gamma}^{\dagger} \boldsymbol{\Phi}_{\omega}^{-1} \boldsymbol{\Gamma}\right) / N$.

\section{Derivation of The Proposed Adaptive Receiver}

In what follows, referring the results of (5), (7), and (11), we present the proposed adaptive receiver processing. From (4) and (5), let us define $\mathcal{Z}=\left[\mathcal{Z}_{1}^{T}, \mathcal{Z}_{2}^{T}\right]^{T}, \mathcal{Y}_{i}=\left[\mathcal{Y}_{i}^{k T}, \overline{\mathcal{Y}}_{i}^{k+1 T}\right]^{T}$ $(i=1,2)$, and $\mathcal{X}=\left[\mathcal{X}_{1}^{k T}, \mathcal{X}_{2}^{k T}\right]^{T}$. Then, stacking $\mathcal{Z}_{1}$ and $\mathcal{Z}_{2}$, the outputs of the self-interference cancellation given by (5) can be expressed as

$$
\mathcal{Z}=\left[\begin{array}{c|c|c}
\mathbf{I}_{2 N} & \mathbf{0}_{2 N \times 2 N} & \boldsymbol{\Sigma}_{1} \\
\hline \mathbf{0}_{2 N \times 2 N} & \mathbf{I}_{2 N} & \boldsymbol{\Sigma}_{2}
\end{array}\right]\left[\begin{array}{c}
\mathcal{Y}_{1} \\
\hline \mathcal{Y}_{2} \\
\hline \mathcal{X}
\end{array}\right]
$$

where $\boldsymbol{\Sigma}_{1}$ and $\boldsymbol{\Sigma}_{2}$ are defined as [see (4)]

$$
\boldsymbol{\Sigma}_{1}=\left[\begin{array}{cc}
-\boldsymbol{\Sigma}_{11} & -\boldsymbol{\Sigma}_{12} \\
\boldsymbol{\Sigma}_{12}^{\dagger} & -\boldsymbol{\Sigma}_{11}^{\dagger}
\end{array}\right], \quad \boldsymbol{\Sigma}_{2}=\left[\begin{array}{cc}
-\boldsymbol{\Sigma}_{21} & -\boldsymbol{\Sigma}_{22} \\
\boldsymbol{\Sigma}_{22}^{\dagger} & -\boldsymbol{\Sigma}_{21}^{\dagger}
\end{array}\right] .
$$

From (7)-(9) and (11), we can see that the combined processing of TR-STBC decoupling and MMSE equalization can be equivalently expressed as

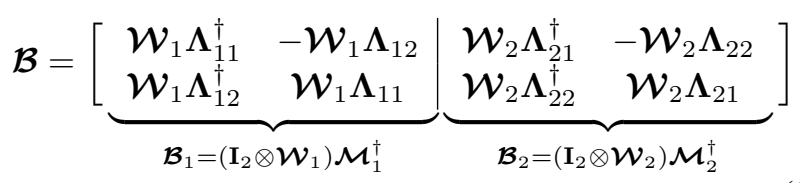

where it is noticed that $\mathcal{B}_{i}(i=1,2)$ is an orthogonal matrix, since $\mathcal{W}_{i}$ is a real-valued diagonal matrix and $\boldsymbol{\Lambda}_{p q}$ is a diagonal matrix. By premultiplying $\mathcal{B}$ to (12), the output of the MMSE equalization for two streams is given by

$$
\left[\begin{array}{c}
\hat{\mathcal{D}}_{1}^{k} \\
\hat{\mathcal{D}}_{2}^{k}
\end{array}\right]=\mathcal{B}_{1} \mathcal{Y}_{1}+\mathcal{B}_{2} \mathcal{Y}_{2}+(\underbrace{\mathcal{B}_{1} \boldsymbol{\Sigma}_{1}+\mathcal{B}_{2} \boldsymbol{\Sigma}_{2}}_{\mathcal{B}_{3}}) \mathcal{X}
$$

From the fact that the sum or product of two orthogonal matrices has an orthogonal structure, we can see that $\mathcal{B}_{3}$ in (15) is also an orthogonal matrix. As a result, using those properties, (15) can be rewritten as in (16), where it is noticed that $\mathcal{F}$ of size $6 N \times 1$ performs jointly the self-interference cancellation, TR-STBC decoupling, and channel equalization. Furthermore, $\mathcal{F}$ can be adaptively updated by using a block version of the recursive least-square (RLS) algorithm as shown in Table. I (on the next page), where $\mathcal{D}_{k}$ denotes the desired symbol vector given by [7]-[9]

$$
\mathcal{D}_{k}=\left\{\begin{array}{l}
{\left[\begin{array}{c}
\mathcal{D}_{1}^{k} \\
\mathcal{D}_{2}^{k *}
\end{array}\right], \text { for training mode }} \\
{\left[\begin{array}{c}
\overline{\mathcal{D}}_{1}^{k} \\
\overline{\mathcal{D}}_{2}^{k *}
\end{array}\right], \text { for decision-directed mode }}
\end{array}\right.
$$

where $\overline{\mathcal{D}}_{i}^{k}(i=1,2)$ denotes the frequency response of the output of the decision device. From Table. I, it is noticed that that the proposed scheme is efficient since $\boldsymbol{\Xi}_{k}$ is a diagonal matrix, i.e., a complex matrix inversion is not required.

\section{Performance Evaluations}

Simulations have been run to demonstrate the performance of the proposed adaptive joint detection. We considered frequency-selective fading channels with uniform delay power profile and QPSK modulation. We assumed that the signalto-noise ratios (SNRs) at the relay and source terminals are identical [4]. It was also assumed that the channel length of each link is 2, i.e., $L_{1}=L_{2}=P_{1}=P_{2}=2$, and $\mathrm{CP}$ of length-1 $(\mu=1)$ is appended [see Section II. B]. The length of a single data block was 64 , and the training block was 


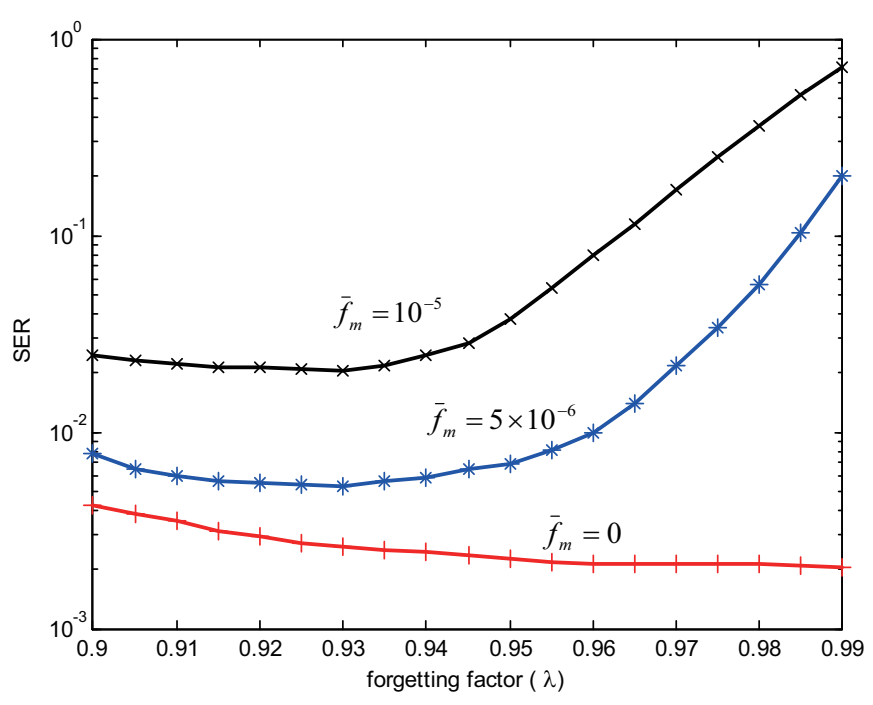

Fig. 2. SER performances of the proposed adaptive receiver as a function of forgetting factor $\lambda(0.90 \leq \lambda \leq 0.99)$, when SNR is $20 \mathrm{~dB}$.

TABLE I

PROCEDURE FOR UPDATING $\mathcal{F}$ USING RLS ALGORITHM [9]

\begin{tabular}{l}
\hline \hline Initialize the algorithm by setting \\
$\qquad \mathcal{F}_{0}=\mathbf{0}_{6 N \times 1}, \quad \mathcal{P}_{0}=\delta \mathbf{I}_{6 N \times 6 N}$ \\
where the regularization parameter $\delta$ is a large positive constant. \\
\hline $\mathcal{F}$ and $\mathcal{P}$ are updated at every two block time $(k=0,2,4, \ldots)$ \\
according to the following recursion \\
$\quad \boldsymbol{\Xi}_{k}=\mathbf{I}_{2 N}+\lambda^{-1} \mathcal{U}_{k} \mathcal{P}_{k} \mathcal{U}_{k}^{\dagger}$ \\
$\mathcal{P}_{k+2}=\lambda^{-1} \mathcal{P}_{k}-\lambda^{-2} \mathcal{P}_{k} \mathcal{U}_{k}^{\dagger} \boldsymbol{\Xi}_{k}^{-1} \mathcal{U}_{k} \mathcal{P}_{k}$ \\
$\quad \mathbf{E}_{k}=\mathcal{D}_{k}-\mathcal{U}_{k} \mathcal{F}_{k}$ \\
$\mathcal{F}_{k+2}=\mathcal{F}_{k}+\mathcal{P}_{k+2} \mathcal{U}_{k}^{\dagger} \mathbf{E}_{k}$ \\
where $\lambda$ is a positive constant close to, but less than 1. \\
\hline \hline
\end{tabular}

appended in every 50 blocks in the proposed adaptive joint detection. In the following results, it is once again noticed that CIRs are constant over single block and it vary every block.

We first evaluate the effect of the forgetting factor $\lambda$ on the symbol error rate (SER) performance and the associated results are shown in Fig. 2. In particular, we considered three different cases for the normalized Doppler frequency ${ }^{1}$, i.e., $\bar{f}_{m}=0 / 5 \times 10^{-6} / 10^{-5}$. Moreover, we restricted our attention to the cases of $0.90 \leq \lambda \leq 0.99$, since $\lambda$ is generally close to 1. From Fig. 2, we can see that the SER performance is degraded as $\lambda$ increases unless the channel is static. On the other hand, when $\bar{f}_{m}=0$ the minimum SER performance could be achieved at $\lambda=0.99$. This is due to the fact that the system can not keep track of the channel variation, when $\lambda$ is very close to 1 .

Fig. 3 depicts the SER performance of the proposed adaptive receiver as a function of link SNR. Based on the results of Fig. 2, we chose $\lambda=0.99 / 0.93 / 0.93$ for the cases of $\bar{f}_{m}=$

${ }^{1} \bar{f}_{m}$ is defined as $\bar{f}_{m}=f_{m} T_{s}$ where $f_{m}$ and $T_{s}$ denote the maximum Doppler frequency and symbol duration, respectively. When $\bar{f}_{m}=10^{-6}$, the channel is considered to be very slow fading channel. On the other hand, $\bar{f}_{m}=10^{-4}$ corresponds to the very fast fading channel [8].

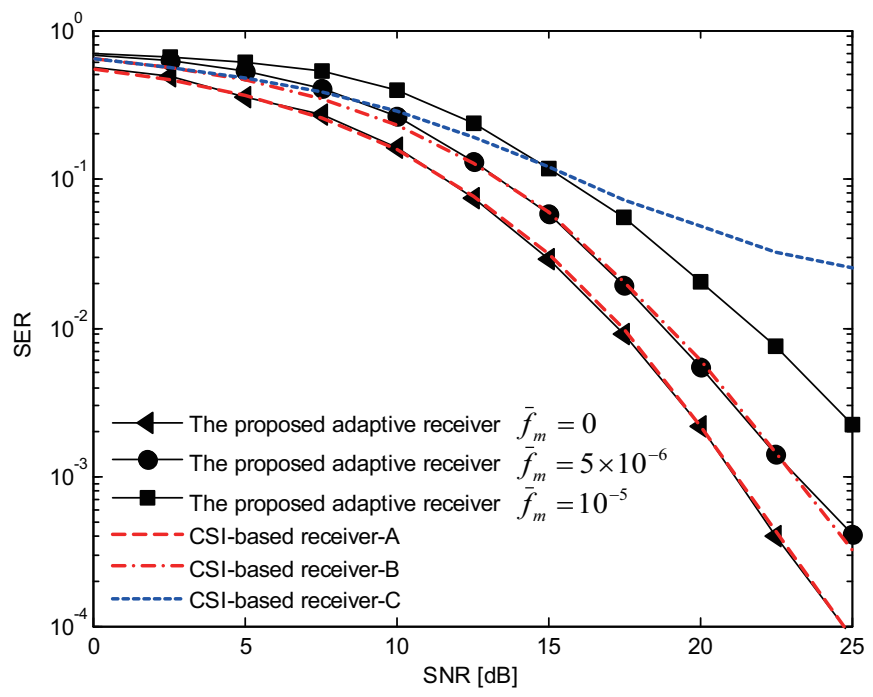

Fig. 3. SER performances of the proposed adaptive receiver and CSI-based receiver-A/B/C

$0 / 5 \times 10^{-6} / 10^{-5}$, respectively.

For comparisons, we considered various CSI-based receivers composing of separate self-interference and MMSE equalization as follows:

- CSI-based receiver-A that has perfect knowledge of CIR and $\sigma_{\Delta_{p}}^{2}$. This receiver provides an ideal performance and cannot be realized in practice.

- CSI-based receiver-B operated based on estimated CIR and perfect $\sigma_{\Delta_{p}}^{2}$, here, we exploit the repeated and phaserotated Chu (RPC) sequences of length- $4 K$ to optimally estimate $\mathrm{CIR}^{2}$ [5]. In practical manner, this receiver cannot be also realized since it requires a perfect knowledge of $\sigma_{\Delta_{p}}^{2}$.

- CSI-based receiver-C that exploits estimated CIR and approximate $\sigma_{\Delta_{p}}^{2}$, here, we approximate $\sigma_{\Delta_{p}}^{2}$ from [6]. This receiver can be used in practice.

The simulation results show that the proposed adaptive scheme approaches the CSI-based receiver-A, when the channel is static $\left(\bar{f}_{m}=0\right)$ by virtue of the convergence property of the RLS algorithm [8]-[9]. On the other hand, the SER performance of the proposed adaptive scheme is degraded as $\bar{f}_{m}$ increases. Nevertheless, compared to the CSI-based receiver- $B$, the proposed scheme provides a better performance in the case of $\bar{f}_{m}=0$ and has a similar performance in the case of $\bar{f}_{m}=5 \times 10^{-6}$. More importantly, it is observed that the proposed scheme outperform the CSI-based receiver$C$ as SNR increases. Basically, the performance degradation of the CSI-based receiver-C is due to the incorrect estimation, i.e., approximation, of $\sigma_{\Delta_{p}}^{2}$. In low SNR regions, the effects of approximation error can be ignored. However, in high SNR regions, it cannot be ignored since its magnitude is much larger than that of noise. Thus, the error floor phenomenon occurs in these regions. To relieve this problem, the additional TS should be inserted at $\mathbb{R}$. Unfortunately, such a procedure makes

\footnotetext{
${ }^{2}$ In the TR-STBC bi-directional relaying protocol considered, the length of TS must be equal to or longer than $4 K$ in order to distinguish all links, where $K=2 \max \left\{L_{1}, L_{2}, P_{1}, P_{2}\right\}-1$
} 
frame structure complex and decreases spectral efficiency. Furthermore, the estimation performance can be improved by using longer TS in the CSI-based receiver-B/C. On the contrary, the proposed joint detection provides better or similar performance with very low overhead.

\section{CONCLUSIONS}

In this paper, we presented the adaptive scheme for use in TR-STBC bi-directional relaying networks. The developed receiver jointly performs the self-interference cancellation, TR-STBC decoupling, and equalization. The main advantage of this receiver is that it requires no CSI, no complex matrix inversion, and a small amount of overhead while delivering a better or similar performance compared to the CSI-based receivers operated with a practical channel estimation.

\section{ACKNOWLEDGEMENT}

This research was funded by the MSIP (Ministry of Science, ICT \& Future Planning), Korea in the ICT R\&D Program 2013

\section{REFERENCES}

[1] B. Rankov and A. Wittneben, "Spectral efficient signaling for half-duplex relay channels," in Proc. of Asilomar Conference on Signals, Systems and Computers, pp. 1066-1071, Oct. 2005

[2] T. Cui, F. Gao, T. Ho, and A. Nallanathan, "Distributed space-time coding for two-way wireless relay networks," IEEE Trans. Signal Process., vol. 57, no. 2, pp. 658-671, Nov. 2009.

[3] N. Xu, and S. Fu, "Performance analysis of space-time codes over twoway relay channels," IEEE Military Communications Conference., vol. 59, no. 8, pp. 4181-4187, Oct. 2010.

[4] F. Gao, R. Zhang, and Y.-C. Liang, "Optimal channel estimation and training design for two-way relay networks," IEEE Trans. Commun., vol. 57, no. 10 , pp. 3024-3033, Oct. 2009.

[5] J. Coon, M. Beach, and J. McGeehan, "Optimal training sequences for channel estimation in cyclic-prefix-based single-carrier systems with transmit diversity," IEEE Signal Process. Lett., vol. 11, no. 9, pp. 729-732, Sep. 2004.

[6] A. S. Lalos, A. A. Rontogiannis, and K. Berberidis, "Frequency domain channel estimation for cooperative communication networks," IEEE Trans. Signal Process., vol. 58, no. 6, pp. 3400-3405, June 2010.

[7] W. M. Younis, A. H. Sayed, and N. Al-Dhahir, "Efficient adaptive receivers for joint equalization and interference cancellation in multiuser space-time block-coded systems," IEEE Trans. Signal Process., vol. 51, no. 11, pp. 2849-2862, Nov. 2003.

[8] J. Coon, M. Sandell, M. Beach, , and J. McGeehan, "Channel and noise variance estimation and tracking algorithms for unique-word based singlecarrier systems," IEEE Trans. Wireless Commun. Lett., vol. 5, no. 6, pp. 1488-1496, Jun. 2006.

[9] S. Haykin, Adaptive Filter Theory, 4th ed., Prentice-Hall, New Jersey, 2002.

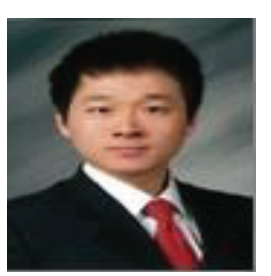

Jeong-Min Choi received B.S. degree in electronic and information engineering from Seoul National University of Science and Technology, Seoul, Korea, in 2010, and the M.S. degree in electrical and electronic engineering from Yonsei University, Seoul, Korea, in 2012, respectively. Since 2012, he has been currently working toward the Ph.D. at the same university. His main research interests include signal processing for digital broadcasting and communication systems.

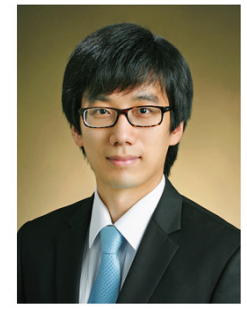

Jae-Shin Han received the B.S. degree in electronics engineering from Konkuk University, Seoul, Korea, in 2011. He is currently pursuing the combined Masters and Doctorate degree in electrical and electronic engineering with the Department of Electrical and Electronic Engineering, Yonsei University, Seoul. His current research interests include channel estimation, equalization, and full-duplex cooperative networks for broadband wireless communication systems

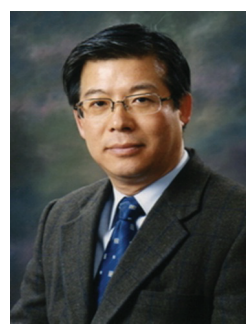

Jong-Soo Seo (F'14) received the B.S. degree in electronics engineering from Yonsei University, Seoul, Korea, in 1975, and the M.S. and Ph.D. degrees from the University of Ottawa, Ottawa, ON, Canada, in 1983 and 1988, respectively. He was with IDC and CAL, Canada, engaged in research on digital satellite communications and data broadcasting systems for six years. Since 1995, he has been with the Department of Electrical and Electronic Engineering, Yonsei University, where he is currently a Professor. Dr. Seo is a Fellow of IEEE and associate editor of the IEEE Transactions on Broadcasting. His current research interests include mobile multimedia broadcasting and beyond fourth-generation mobile radio systems 